\title{
Absolute pitch in blind musicians
}

\author{
Roy H. Hamilton,',2 Alvaro Pascual-Leone' and Gottfried Schlaug, ${ }^{\mathrm{I}, \mathrm{A}}$ \\ 'Department of Neurology, Beth Israel Deaconess Medical Center, 330 Brookline Avenue, Palmer I, Boston, MA 02215; ${ }^{2}$ Harvard Medical School, \\ Boston, MA, USA \\ ${ }^{\text {CAC} C o r r e s p o n d i n g ~ A u t h o r: ~ g s c h l a u g @ b i d m c . h a r v a r d . e d u ~}$
}

Received 8 January 2004; accepted 9 January 2004

DOI: 10.1097/0I.wnr.0000118981.36602.90

\begin{abstract}
Absolute pitch (AP) is possessed by only a small percentage of musicians (typically $<20 \%$ ). From a sample of 46 early blind subjects, we identified 21 who had musical training, 12 of whom (57.1\%) reported having AP, reflecting markedly increased prevalence compared to sighted musicians, despite the fact that mean age of commencement of musical training was significantly later among blind than sighted AP musicians in our database. MR images
\end{abstract}

acquired in a subset of blind AP musicians revealed greater variability in planum temporale asymmetry compared with the increased left-sided asymmetry previously described in sighted AP musicians. This suggests that neural mechanisms underlying AP in blind musicians could differ from those in sighted musicians. NeuroReport 15:803-806 (c) 2004 Lippincott Williams \& Wilkins.

Key words: Absolute pitch; Morphometry; Musicians; Blind; Planum temporale

\section{INTRODUCTION}

Absolute pitch (AP) is defined as the ability to identify a particular pitch of the Western musical scale without any external reference tone [1-3]. AP is present in a minority of trained Western musicians [4]. The prevalence of AP in the general population has been estimated as $1 / 1500-1 / 10000$ [3]. Several studies into the role of musical training have demonstrated that $\mathrm{AP}$ is more likely to develop if the commencement of training is early [4-7]. A number of investigators reported that their groups of AP subjects had a typical age of commencement of $5 \pm 2$ years while non-AP musicians typically started 1-2 years later. Even among early-trained musicians, however, AP occurs in a minority of individuals, sometimes with a higher than expected familiar incidence, suggesting that there are additional factors (e.g. particular anatomical brain features and genetic traits) that may contribute to the AP phenotype.

Although the neural underpinnings of AP remain unclear, recent neuroimaging studies have elucidated functional and anatomical brain correlates to AP [7-11]. A growing body of data indicates that there are significant differences in brain morphology between sighted musicians with AP and those without AP in the planum temporale (PT), a region traditionally associated with language and auditory processing. Sighted individuals with AP show an exaggeration of the normal leftward asymmetry of the PT seen in both righthanded non-AP musicians and non-musicians [8,11]. Recent evidence suggests that the basis of this increased asymmetry is a significant reduction in the size of the right PT with a non-significant trend for a larger left PT [7]. Recent functional imaging studies showed activation of the left more than the right PT in musical tasks in AP musicians, not seen in non-AP subjects $[10,11]$. Since the prevalence of AP among Anglo-American musicians is relatively low, there has been an interest in examining whether AP develops more frequently among other groups. Several studies have reported a 2- to 3-fold higher incidence of AP musicians in Asian countries [2] and among Asian Americans [4]. Other studies examining relationships between autistic children and AP suggest that the prevalence of AP among people with autism could be as high as 1 in 20 (5\%) [12]. Patients with Williams syndrome also seem to have a higher incidence of AP than one would expect to find in the normal population [13]. The observation that many blind musicians achieve very high levels of musical proficiency has lead to the popular impression that blindness may be associated with special auditory perceptual skills. Indeed, there is evidence that early blind subjects are able to localize sound sources better than sighted subjects $[14,15]$ and that in doing so they engage parietal and occipital lobe structures [16-18]. In the present study, we surveyed a group of blind subjects to determine the incidence of AP among those who had musical training. We also sought to determine whether early age at onset of musical training or interhemispheric asymmetry of the PT seen in sighted AP musicians play a critical role in the development of $\mathrm{AP}$ among the blind.

\section{MATERIALS AND METHODS}

Subjects: A total of 46 early blind individuals was recruited on a voluntary basis from regional schools for the blind for previous studies unrelated to musical aptitude, musical exposure, or AP ability. This group was then screened for musicianship, and twenty-one (nine males, 12 females; mean age 47.4 years) were either professional or 
Table I. Right, left, and combined PT surface areas and asymmetry coefficients (mean \pm s.d.) in blind and sighted absolute pitch musicians and sighted nonmusicians.

\begin{tabular}{lcccc}
\hline & Right PT & Left PT & Total PT & $\delta$ PT \\
\hline AP blind & $909.02 \pm 223.67$ & $1106.23 \pm 291.83$ & $2015.25 \pm 332.28$ & $-0.18 \pm 0.39$ \\
Non-AP blind & $852.53 \pm 134.66$ & $1225.52 \pm 132.41$ & $2078.06 \pm 203.34$ & $-0.36 \pm 0.17$ \\
AP sighted $^{\mathrm{a}}$ & $828.17 \pm 232.61$ & $1401.55 \pm 444.71$ & $2229.72 \pm 606.89$ & $-0.50 \pm 0.27$ \\
Non-AP sighted $^{\mathrm{a}}$ & $1079.02 \pm 212.18$ & $1333.82 \pm 285.84$ & $2412.84 \pm 461.02$ & $-0.21 \pm 0.16$ \\
Non-musician sighted $^{\mathrm{a}}$ & $1043.00 \pm 283.07$ & $1365.01 \pm 342.73$ & $2408.01 \pm 388.01$ & $-0.28 \pm 0.33$ \\
\hline
\end{tabular}

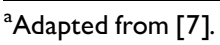

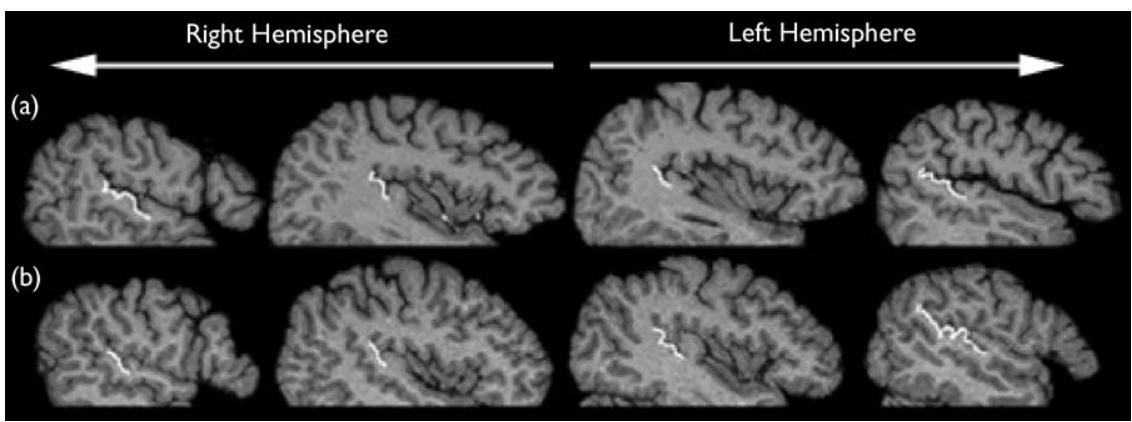

Fig. I. Two blind subjects with AP. Subject (a) had a rightward PT asymmetry, subject (b) had an increased leftward PTasymmetry. The PT is marked in white on two sagittal slices representing right and left hemisphere (see $[8,20]$ for details on PT delineation).

amateur musicians. The remaining blind subjects did not have any musical training. All 21 subjects were Caucasian, blind due to peripheral causes, and had acquired blindness before age 6 . None had any residual vision (absolute blindness). All of the subjects gave written informed consent to the study, which was approved by the institutional review board. Subjects were administered a questionnaire that queried various aspects of their musical experience and the presence or absence of AP. Although several groups have reported a high agreement between self-report of AP and performance on AP tests $[1,4,7,19]$, we confirmed the presence of AP using two established tests in a subset of 7 subjects who reported AP and agreed to be tested. Other subjects who reported AP were unavailable for formal testing. The first test consisted of 13 sine wave tones taken from the chromatic scale (F\#3 to $\mathrm{F} \# 4$ ), repeated randomly four times for a total of 52 tones. The second AP test consisted of 12 sine wave tones taken from $41 / 2$ octaves (F2 to G5), separated by musical fourths (F2, Bb2, Eb3, etc.), presented four times in random order for a total of 48 tones. In each task, subjects listened to the complete set of tones twice and were permitted to make corrections as they saw fit. Responses that were within one semitone difference from the presented tone were regarded as correct $[2,19]$, and $90 \%$ accuracy on both tests was set as a cutoff for the determination of AP ability (see [7] for more details on these tests). All seven of the blind musicians tested who had claimed to have absolute pitch were confirmed by these criteria, thus confirming the validity of our AP questionnaire.

MR data acquisition and morphometric analysis: We were able to obtain high resolution anatomical scans on a total of 13 subjects. A subset of eight blind subjects with selfreported AP and five blind subjects without $\mathrm{AP}$ underwent high-resolution magnetic resonance imaging. The eight self- reported AP subjects who were imaged included all seven subjects who had undergone pitch testing (see above) and one who had not due to scheduling conflicts. Images were acquired using a T1-weighted magnetization prepared, rapid acquisition gradient-echo (MPRAGE) pulse sequence on a $1.5 \mathrm{~T}$ Siemens Vision MR system with echo-planar imaging capability (Siemens Medical Systems, Erlangen, Germany). The image dataset had a voxel resolution of $1.0 \mathrm{~mm}^{3}$.

The anatomical boundaries of the PT were defined according to previously published criteria $[7,11,20]$. The PT contours on sagittal slices were represented as splines in order to approximate the curvature found in the gyral pattern of the brain [20]. A triangular mesh was generated between contours on consecutive slices, and the surface area was then calculated as a sum of the area of all the triangles defining the surface of interest. Left-right asymmetry of the PT surface area was expressed as the asymmetry coefficient $\delta P T(R-L /(R+L) \times 0.5)$ with $\mathrm{R}$ and $\mathrm{L}$ representing the surface areas of the right and left PT. Negative values indicate leftward asymmetry; positive values indicate rightward asymmetry (Table 1; Fig. 1).

\section{RESULTS}

In our sample of 21 blind musicians $12(57.1 \%)$ reported having AP. The accuracy of subject self-reporting of AP was confirmed in a subset of seven of these 12 subjects using established tests. In comparison to a group of sighted musicians previously tested in an identical fashion [7], the prevalence of AP seen in blind musicians was significantly elevated $\left(\chi^{2}=21.60, \alpha<0.001\right)$. Interestingly, the age of commencement of musical training among the blind AP musicians (average 8.0 years; range 3-14 years) was significantly $(t=3.93, p<0.001)$ later (three subjects at age 8 , two at 9 , one at 10 , one at 14) than that which had been 
observed among sighted AP musicians (average 5.2 years; range 3-7 years) taken from our database of AP musicians.

Blind AP musicians (unselected for handedness) did not demonstrate the same increased planum temporale asymmetry (mean ( \pm s.d.) $\delta \mathrm{PT}=-0.18 \pm 0.39$; range -0.98 to 0.25 ) previously observed in sighted AP musicians (mean $\delta \mathrm{PT}=-0.50$; range -1.04 to 0.06 ). In contrast, blind $\mathrm{AP}$ musicians showed a much greater degree of variability in $\delta \mathrm{PT}$ (Fig. 1) than had been previously demonstrated in sighted AP musicians, non-AP musicians, and non-musicians [7]. Furthermore, blind AP musicians demonstrated greater variability of PT asymmetry than blind non-AP musicians in our subject population (mean $\delta \mathrm{PT}=$ $-0.36 \pm 0.17$; range -0.58 to -0.11 ) (Table 1 ).

\section{DISCUSSION}

This study builds upon a body of prior evidence in which key characteristics of AP in sighted patients have been convincingly demonstrated. The results of this study suggest that there are at least three major differences in the phenomenology of AP between sighted and blind musicians: (1) increased prevalence of AP among blind musicians, (2) later onset of musical training among blind AP musicians, and (3) increased variability of PT asymmetry among blind AP musicians.

The highest reported prevalence of AP among Caucasian musicians is $\sim 18 \%$. For reasons that are yet to be fully explained, the prevalence of AP among Asians is higher [4]. Our finding of a $57.1 \%$ prevalence of AP among a group of blind Caucasian musicians is therefore remarkable. Furthermore, these results add to a growing body of data regarding the interactions between environmental and presumed genetic factors in the development of AP. Studies demonstrating increased prevalence of AP within families [4], and observations from twins studies [21] suggest a heritable component to pitch discrimination ability. Since all of the blind musicians in our study were rendered blind by peripheral causes that occurred after birth, the high prevalence of $\mathrm{AP}$ in these subjects indicates that an environmental stimulus such as the loss of sight can somehow facilitate the development of $\mathrm{AP}$, leading to a higher number of AP in this group then would be expected based on the incidence in sighted groups. In previous studies among sighted subjects, AP was found to most likely manifest itself during a critical period of development early in life, during which time exposure to music was a prerequisite [4-7]. In blind subjects however, it seems that an early age of commencement of musical training does not play as critical a role. Even in our small sample of blind subjects, several individuals with AP had not initiated musical training until their late childhood or adolescence, which is unusually late compared to sighted individuals with AP. This suggests that in blind subjects, even late onset of musical training serves to induce the neural changes that may underlie the development of AP. It is tempting to hypothesize that the plastic changes induced in the brain by blindness [16-18] contribute to this fact. Finally, among sighted subjects with AP, PT asymmetry has been shown to be a critical factor in the development of AP $[7,8,11]$. Our preliminary morphometric data suggests that the increased leftward asymmetry of the PT that has been robustly demonstrated in sighted AP musicians is not a necessary feature of AP among the blind. Some subjects were extremely lateralized to the left, others to the right, and some did not differ from sighted non-musician control groups. Certain methodological limitations to the current study should be addressed. One important caveat is that, due to the limited size of our subject pool of early peripherally blind musicians, subjects were unselected for handedness. Although blind subjects' degree of PT asymmetry did not correlate with handedness in this small subject population, a larger sample of patients should be tested in order to determine whether handedness has the same correlation with PT asymmetry indices in blind subjects as it does in sighted subjects. In addition, hand preference and hand dominance may be differently distributed in blind subjects compared to sighted subjects [22]. In this context it is important to note that hand dominance in early blind subjects is a notably unreliable measure that may depend on the tasks explored [23]. A second methodological limitation is that pitch testing was not pursued among blind musicians who denied having AP or among blind non-musicians. The correlation between selfreporting of $\mathrm{AP}$ and the presence of $\mathrm{AP}$ has in prior literature been shown to be very high $[3,4,7,8]$, and there is little reason to suspect that experienced musicians, blind or sighted, would be unaware of their own AP ability. If, however, this were not the case for blind musicians who denied having $\mathrm{AP}$, the actual prevalence of $\mathrm{AP}$ among the blind may have actually been underestimated. It is also plausible that blind persons irrespective of musicianship generally have better pitch processing abilities, and that the increased prevalence of AP observed in our study reflects an overall increase in AP among blind individuals compared with the sighted. Because AP in non-musicians is extremely rare, a very large survey of blind non-musicians would have to be undertaken to answer this question definitively. Such a finding would further support our hypothesis that fundamental epidemiologic and anatomic differences exist between blind and sighted subjects with regard to AP ability. Taken together, the findings of increased prevalence of AP among blind musicians, their later age at commencement of musical training, and the lack of a consistently leftward PT asymmetry suggests that $\mathrm{AP}$, at least in some blind musicians, could manifest itself through a different mechanism than in the sighted. A growing body of evidence indicates that early blind subjects are able to recruit the visually deafferented occipital cortex for non-visual tasks, including auditory information processing [16-18]. This cross-modal plasticity may provide an additional substrate for the development of AP in the blind compared with the sighted, which could account for the higher than expected prevalence and prolonged critical period of AP development in the blind. The ability to perceive auditory stimuli as belonging to categories is one of the features that differentiates AP musicians from individuals without AP [24]. Recent evidence demonstrates that visual association areas are intimately involved in the processing of categorical visual information [25]. In the absence of sight, these visual association areas may facilitate categorization of auditory information. Such cross-modal cortical plasticity may at least partially account for our finding that blind subjects with AP are not as dependent upon the same morphologic changes in the auditory cortex that correlate with AP ability among the sighted. 


\section{CONCLUSIONS}

While this study is limited in its scope, and does not establish a specific mechanism for the acquisition of $\mathrm{AP}$ in blind musicians, our results argue that $\mathrm{AP}$ among the blind is not due to an enhancement of the same determinants that are critical among the sighted: early musical training and increased PT asymmetry. The notion that cross-modal plasticity involving the occipital cortex may provide an additional neural substrate for the development of AP in the blind is an intriguing hypothesis that merits further investigation. It would be informative for future studies to replicate the current findings in larger populations of blind musicians as well as to further investigate the anatomic and functional underpinnings of AP in blind and sighted musicians.

\section{REFERENCES}

1. Deutsch D (ed.) The Psychology of Music. New York: Academic Press; 1982.

2. Miyazaki K. Musical pitch identification by absolute pitch possessors. Percept Psychophys 44, 501-512 (1988).

3. Takeuchi AH and Hulse SH. Absolute pitch. Psych Bull 113, 345-361 (1993).

4. Gregersen PK, Kowalsky E, Kohn N and Marvin EW. Absolute pitch: prevalence, ethnic variation, and estimation of the genetic component. Am J Hum Genet 65, 911-913 (1999).

5. Seargeant D. Experimental investigation of absolute pitch. J Exp Psychol 103, 37-44 (1969).

6. Krumhansl C. Music psychology: tonal structures in perception and memory. Annu Rev Psychol 42, 277-303 (1991).

7. Keenan JP, Thangaraj V, Halpern AR and Schlaug G. Absolute pitch and planum temporale. Neuroimage 14, 1402-1408 (2001).

8. Schlaug G, Jancke L, Huang Y and Steinmetz H. In vivo evidence of structural brain asymmetry in musicians. Science 267, 699-701 (1995).

9. Zatorre RJ, Perry DW, Beckett CA, Westbury CF and Evans AC. Functional anatomy of musical processing listeners with absolute pitch and relative pitch. Proc Natl Acad Sci USA 95, 3172-3177 (1998).
10. Ohnishi T, Matsuda H, Asada T, Aruga M, Hirakata M, Nishikawa M et al. Functional anatomy of musical perception in musicians. Cerebr Cortex 11, 754-760 (2001).

11. Schlaug $G$. The brain of musicians. A model for functional and structural adaptation. Ann NY Acad Sci 930, 281-299 (2001).

12. Brown WA, Cammuso K, Sachs H, Winklosky B, Mullane J and Bernier R. Autism-related language, personality, and cognition in people with absolute pitch: results of a preliminary study. J Autism Dev Disord 33, 163167 (2003).

13. Lenhoff HM, Perales $O$ and Hickok G. Absolute pitch in Williams syndrome. Music Percept 18, 491-503 (2001).

14. Lessard N, Pare M, Lepore F and Lassonde M. Early-blind subjects localize sound sources better than sighted subjects. Nature 395, 278-280 (1998).

15. Roeder B, Teder-Sälejárvi W, Sterr A, Rösler F, Hillyard SA and Neville HJ. Improved auditory spatial tuning in blind humans. Nature 400, 162166 (1999).

16. Weeks R, Horwitz B, Aziz-Sultan A, Tian B, Wessinger M, Cohen LG et al. A positron emission tomography study of auditory localization in the congenitally blind. J Neurosci 20, 2664-2672 (2000).

17. Kujala T, Kimmo $A$ and Näätänen R. Cross-modal reorganization of human cortical functions. Trends Neurosci 23, 115-120 (2000).

18. Rauschecker JP. Compensatory plasticity and sensory substitution in the cerebral cortex. Trends Neurosci 18, 36-43 (1995).

19. Zatorre RJ and Beckett C. Multiple coding strategies in the retention of musical tones by possessors of absolute pitch. Mem Cogn 17, 582-589 (1989).

20. Steinmetz H, Volkmann J, Jancke L and Freund HJ. Anatomical left-right asymmetry of language-related temporal cortex is different in left- and right-handers. Ann Neurol 29, 315-319 (1991).

21. Drayna D, Manichaikul A, de Lange M, Sneider H and Spector T. Genetic correlates of musical pitch recognition in humans. Science 291, 1969-1972 (2001).

22. Corballis MC and Beale IL. The Ambivalent Mind: The Neuropsychology of Laterality. Chicago: Nelson Hall; 1983.

23. Millar S. Is there a "best hand" for Braille? Cortex 20, 75-87 (1984).

24. Rakowski A. Categorical perception in absolute pitch. Arch Acoust 18, 515-523 (1993).

25. Spiridon M and Kanwisher N. How distributed is visual category information in human occipito-temporal cortex? An fMRI study. Neuron 12, 1157-1165 (2002).

Acknowledgements: We would like to thank Demma Rodriguez for her involvement in subject testing and Marc Ruel for critically reading a previous version of this manuscript. This study was supported by a grant from the International Foundation for Music Research (IFMR) and in part by grants from the National Science Foundation (BCS-0132508), National Eye Institute (EYI209I), National Institute of Mental Health (MH57980, MH60734), and the General Clinical Research Center at Beth Israel Deaconess Medical Center (National Center for Research Resources MOI RROI032). G.S. is also supported in part by a Doris Duke Charitable Foundation Clinical Scientist Development Award. 